\title{
A Fair Service Work Scheduling Approach for Differentiated Services in Optical Access Networks
}

\author{
NamUk Kim, HyunHo Yun, and Minho Kang \\ Optical Internet Research Center, Information and Communications University, 58-4, \\ Hwaam-Dong, Yuseong-gu,Daejeon, 305-732, Korea \\ \{niceguy, exhyho, mhkang\}@icu.ac.kr
}

\begin{abstract}
We address the QoS (Quality of Services) bandwidth reservation and service work scheduling mechanism of how to fairly provide advance quality of service in polling based TDM networks like Ethernet PON (Passive Optical Networks). Unlike existing differentiated QoS supporting schemes, such as SP (Strict Priority) based DBA(Dynamic Bandwidth Allocation) or static work scheduling with min-max theory, proposed service quality pre-engagement (SQP) for prioritized packet scheduling and equal weighted fair bandwidth arbitration (EWF-BA) for fair services among access nodes reflect the dynamically changing service pattern of access node like ONU (Optical Network Unit). The performance results show that the service fairness and QoS are tightly coupled and proposed mechanisms achieve better QoS performances with high fairness and better network performances both in Poisson and Selfsimilar traffic environment.
\end{abstract}

\section{Introduction}

The polling based TDMA over the shared medium is recently adopted to many access networks such as Ethernet-PON (Passive Optical Network) and WDM optical access networks[1][2]. Especially, the upstream multiple access control is important because upstream channel must be shared efficiently and fairly by ONUs without channel collision while both high performances and relative QoS with differentiated services among classes are guaranteed. These objectives are mainly achieved by the service work scheduling, the controlled bandwidth allocation mechanism of OLT (Optical Line Termination), and the packet scheduling of ONU, generally the strict priority (SP) queuing or weighted fair queue (WFQ) service[4]. Up to now, some approaches, mainly be categorized into static bandwidth allocation (SBA) and DBA, are contributed to this topic for the optimized QoS performances[2]. These mechanisms guarantee the minimum service fairness with fixed service slot or min-max theory based dynamic slot allocation and provide high channel utilization, especially in DBA. But because these targets for the fairness of service slot not service thread, consistent service pattern of ONU, it restarts a new cycle although the unused bandwidth currently exists, which 
can be used for urgent heavy loaded ONUs. This can affect the fairness and effectiveness of services in short time epoch.

In this paper, we propose SQP which applies QoS bandwidth prediction and prereservation scheme based on SP. Up to now, most proposed scheduling methods only consider the prioritized services separated from DBA specific operation but we converge them for supporting efficient differentiated services in intra ONU. Then, we introduce EWF-BA, the policy based service work scheduling, to get the thread based pipeline service for high QoS performances as well as efficient service fairness in inter ONU domain. Our proposed schemes can be adapted to all kinds of generalized polling based TDMA network independent from physical media and topological difference.

\section{The Fair Differentiated Services Supporting with SQP and EWF-BA}

The SP can support QoS guaranteed services regardless of traffic changes but the bandwidth is easily monopolized by heavy traffic loaded high priority classes. Weight based methods like WFQ or DRR can not guarantee good performances by variable Ethernet frame size and high complexity[3][4]. Proposed SQP-DBA is a modified DBA mechanism based on SP scheduling and QoS bandwidth prereservation. For easy expressions, following notations for class i traffic are used when $O N U_{j}$ and $m_{t h}$ cycle are assumed.

N,K: Number of ONUs and traffic classes. $D_{i}^{m}$ : Reported bandwidth for next cycle. $S_{i}^{m}$ : Total serviced work(bandwidth).
$Q_{i}^{m}(t)$ :Queue size at time $\mathrm{t}$.

$A_{i}^{m}:$ Allocated bandwidth.

$V_{l}$ : Transmission speed of link.

We assumed that traffic is grouped into $\mathrm{K}$ service classes, which are ordered, such that class $\mathrm{i}$ is better than class $\mathrm{i}+1$ for $0<i<K-1$, in terms of the queuing delay and packet losses. Generally, it is possible to assume that the size of class0 queue of $O N U_{j}, Q_{0, j}\left(t_{m}\right)$, where $t_{m}$ is $m_{t h}$ service slot instance, does not rapidly change during several frame cycles because the aggregated CBR and real time application traffic shows nearly constant arrival pattern although their frame size varies. Based on this, in SQP-DBA, ONU reserves the service slot of next cycle as not $Q_{0, j}\left(t_{m}+A_{j}^{m} / V_{l}\right)$ but $Q_{0, j}\left(t_{m}+A_{j}^{m} / V_{l}\right)+$ QoS reservation bandwidth which is same size of current cycle's class0 serviced work. In addition, we assume the gated service scheme to minimize the long switching-over time between inter-classes and bandwidth monopolization of higher classes. Hence, the final report queue size and final service work is different to each class as follows.

$$
\begin{aligned}
& D_{0, j}^{m}=\mathrm{Q}(\text { late arrival })+\mathrm{Q}(\mathrm{QoS} \text { reservation })=Q_{0, j}^{m}\left(t_{m}+\sum_{i=0}^{K-1} A_{i, j}^{m} / V_{l}\right)+S_{0, j}^{m} \\
& D_{0, j}^{m}(i \neq 0)=\mathrm{Q}(\text { late arrival or unfinished work })=Q_{i, j}^{m}\left(t_{m}+\sum_{i=0}^{K-1} A_{i, j}^{m} / V_{l}\right) \\
& A_{0, j}^{m}=\min \left(Q_{0, j}^{m}\left(t_{m}\right), A_{j}^{m}\right)=S_{0, j}^{m}, A_{l o w, j}^{m}=A_{j}^{m}-\min \left(Q_{0, j}^{m}\left(t_{m}\right), A_{j}^{m}\right) \\
& S_{i+1}^{m}=\min \left(Q_{i+1, j}^{m}\left(t_{m}\right), A_{j}^{m}-\sum_{n=0}^{i} Q_{N, j}^{m}\left(t_{m}\right), \text { when } i<K-2 .\right.
\end{aligned}
$$


Because the arrival pattern of lower priority ones such as aggregated VBR and nrt-VBR traffic rapidly changes, the efficiency of QoS bandwidth pre-reservation is relatively low. In this reason, we apply the general DBA's three step channel reservation mechanism to lower priority classes. With this, the high channel utilization of SQP-DBA is maintained by DBA and service quality reservation is done by SQP. Therefore, the QoS performance enhancement is expected to every class within the allocated bandwidth. But SQP client has no control authority to bandwidth allocation and its algorithm targets for QoS based differentiated services only in ONU. Hence, there must be a optimized inter ONU service scheduling algorithm in OLT, which is defined as bandwidth arbitration in this paper, for high QoS performances in network domain. General service work scheduling, SBA or DBA based, targets for the minimum service fairness by set limitation on slot size. However, these do not consider the service pattern of ONU, which can be important to QoS guaranteeing as well as network performances in short time epoch under heavy traffic environment. The service fairness shows how fairly and efficiently bandwidth is distributed to ONUs in long and even in short time period for full services. Because upstream packet service is done under non-exhaustive vacation service scheme, fairness is directly determined by two elements, $T_{j}^{m}$ and $A_{j}^{m}$ and these are tightly coupled with each other by arbitration. Proposed EWF-BA is a new service balancing mechanism that arbitrates the service slot size with real time information induced from traffic demands as well as the service thread of each ONU. The main idea is as follows. When the ideal cycle frame under SLA is limited to $T_{c}$ excluding guard band, OLT gets three kinds of information in every arbitration points, sequentially expressed as $\sum_{n=0, n \neq j}^{N-1} A_{n}^{m}, D_{j}^{m}$, and $T_{c}^{m} V_{l}$. To effectively reflect the service thread or allocation pattern to $A_{j}^{m}$, we introduce two parameters. The one is the bandwidth occupation ratio $C^{m}$. It shows the serviced work ratio of each ONU normalized to total serviced work of ONUs in previous cycle and expressed as follows when $t_{j, R}^{m}$ is the $m_{t h}$ is the reporting instance.

$$
C_{j}^{m+1}=A_{j}^{m+1} / \sum_{n=0}^{N-1} A_{n}{ }^{m+1}=A_{j}^{m+1} /\left(t_{j, R}^{m+1}-t_{j, R}^{m}\right) V_{l}, 0<j<N-1 .
$$

$C^{m}$ can be easily calculated from DBA operation. The other parameter is the bandwidth overflow ratio, $R_{j}^{m}=\left[\left(\sum_{n=0, n \neq j}^{N-1} A_{n}{ }^{m}+D_{j}^{m}\right)-T_{c} V_{l} / T_{c} V_{l}\right]$, which is the excessively requested bandwidth ratio normalized by the SLA threshold value. It is meaningful only when the sum of service reserved and current demanding work is greater than $T_{c} V_{l}$. As mentioned, the over-reserved traffic service seriously degrades QoS performances and service fairness by the long service interval and bandwidth monopolization. To avoid these problems, OLT proportionally reflects the occupation ratio $C_{j}^{m}$ to the final reduction weight value, $W_{j}^{m+1}$, while the thread of each ONU is kept as much as possible. OLT calculates final $A_{j}$ with the reduction weight calculated from the occupation ratio and the overflow ratio as follows.

$$
W_{j}^{m}=C_{j}^{m} R_{j}^{m}=A_{j}^{m} N\left[\left(\sum_{n=0, n \neq j}^{N-1} A_{n}{ }^{m}+D_{j}^{m}\right)-T_{c} V_{l} / T_{c} V_{l} \sum_{n=0}^{N-1} A_{n}{ }^{m}\right]
$$


if $\sum_{n=0, n \neq j}^{N-1} A_{n}{ }^{m}+D_{j}^{m}>T_{c} V_{l}, A_{j}^{m+1}=D_{j}^{m}-D_{j}^{m} C_{j}^{m} R_{j}^{m}=D_{j}^{m}\left(1-W_{j}^{m}\right)$.

else, $A_{j}^{m+1}=D_{j}^{m}$ by general DBA scheme.

Therefore, In EWF-BA, by the consideration of individual ONU's service thread in near real time, greedy ONU that has monopolized large bandwidth previously receives the reduced bandwidth with the same reduction weight to pervious occupation ratio in current cycle. The light loaded ONU rarely experiences arbitration effects due to low occupation ratio. It is efficient for minimum bandwidth guaranteeing under burst traffic environment. At the same time, because the heavy traffic load of some ONUs is efficiently distributed to every ONUs, There is no serious performance degradation of some ONUs. Moreover, the fairness guaranteed QoS service can be achieved since occupation ration which shows service pattern is effectively reflected to reduction weight and the cycle frame does not dynamically change by this. Below figure 1 shows the overall procedure of EWF-BA.
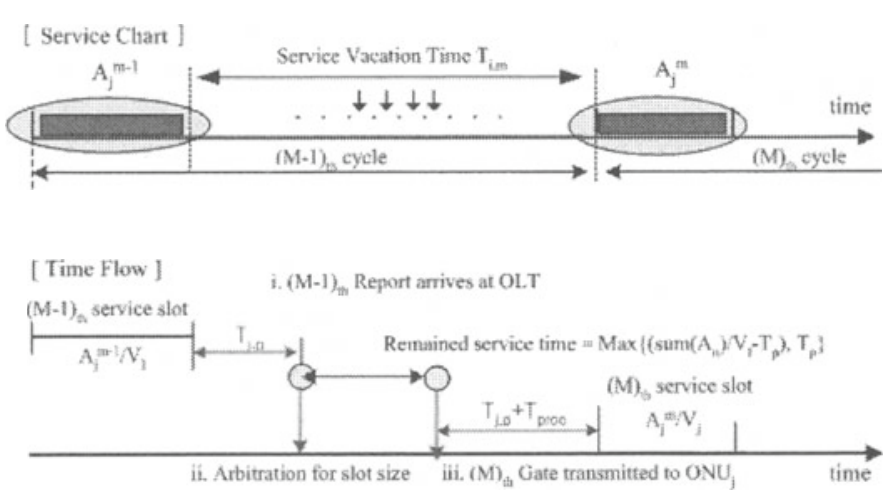

Fig. 1. Service work scheduling mechanism for EWF-BA.

\section{Simulation Results and Performance Evaluation}

For effective simulations, we assume that all incoming frames are classified into three traffic service classes in ONU as class0 for the CBR traffic and real time, class1 for non-real time and class2 for best effort service. The ON/OFF model is applied to the generation of Poisson and burst traffic and load is uniformly distributed over three classes. The frame size distribution follows that of the real traffic model in both Poisson and self similarity burst traffic analysis model $[6,11]$. In burst traffic environment, we applied Pareto distributed(Hurst parameter $0.8,0.9)$ traffic generation scenario. 

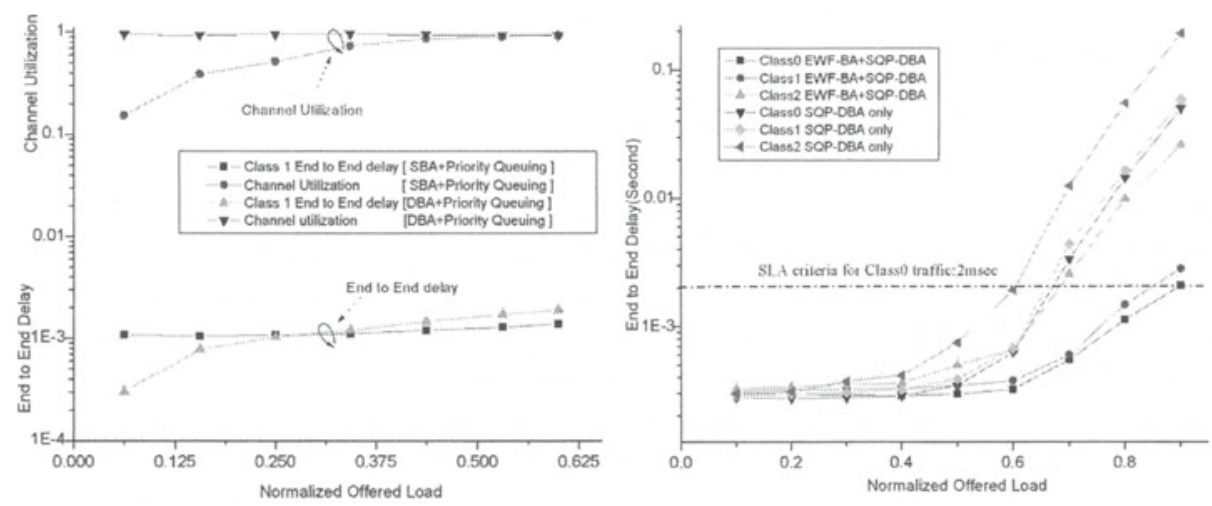

Fig. 2. (a)Average end to end delay and utilization of each class when Poisson traffic applied (b) Average end to end delay when Pareto traffic applied.
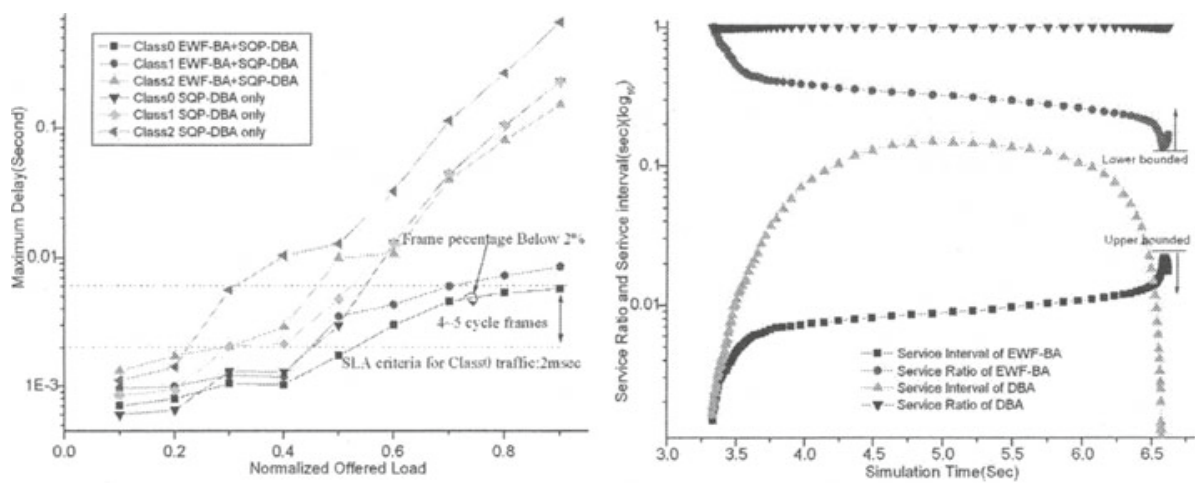

Fig. 3. (a)Maximum end to end delay of each class when Pareto traffic applied.(b) Service ratio and interval when Pareto traffic applied $(\mathrm{load}=0.8, \mathrm{H}=0.9)$.

Figure 2(a) shows DBA achieves better utilization and lower delay in low traffic environment. But its delay gradually increases in high traffic environment due to the cycle frame change. Figure 2(b) shows that average delay of SQP-DBA plus EWF-BA is more enhanced than SQP-DBA only case in every classes. This result comes from the fact that EWF-BA efficiently distributes the heavy traffic load to all ONUs with the equal weighted reduction ratio until network goes into the stable service state. Moreover, figure 3(a) shows that the maximum delay of EWF-BA plus case is smaller than SQP-DBA only case and total numbers of longer delayed frames over targeted SLA value is relatively small. Finally, the congestion resolution period, time gap to overcome burst traffic, is just several cycle frames and this means that EWF-BA can service efficiently heavy loaded 
ONUs while service fairness is maintained among ONUs. This is more obvious in figure 3 (b). This result is derived from the same simulation scenario of above one but more burst environment. In DBA case, the service ratio that means serviced work normalized by the original demand bandwidth is always one by the DBA mechanism. But the service interval is too large to efficiently support the QoS guaranteed services. Moreover, the serious congestion duration is unpredictable and uncontrollable. Oppositely, EWF-BA guarantees the relatively short service interval and its value shows upper bounded pattern. This is possible by the load distribution and the service arbitration of that. The service ratio is generally lower than DBA but is also lower bounded. Therefore, the differentiated services for QoS can be guaranteed within minimum delay by SQP and under stable service pattern and fairness by EWF-BA. In this simulations, the average queue size of EWF-BA is generally lower than DBA but maximum queue size in short epoch is about 1.3 times larger than DBA case in very heavy traffic environment. This is the endurable performance degradation but there must be more improvement to this problem.

\section{Conclusion}

In this paper, we propose the SQP-DBA and EWF-BA for supporting QoS guaranteed fair service under the service work scheduling and reservation. The SQP-DBA achieves the minimum end-to-end delay of the highest priority traffic and high utilization with the minimum effects to lower classes. When EWF-BA scheme is applied, the network performances, especially the delay and service fairness among access nodes, are enhanced even if burst traffic happens in some ONUs. The bandwidth starvation by some heavy loaded ONUs can be also resolved within relatively short time epoch by the EWF-BA's thread based load distribution scheme although more buffer size is needed.

Acknowledgement. This work was supported in part by the Korea Science and Engineering Foundation (KOSEF) through OIRC project and ETRI.

\section{References}

1. Kramer, G., Mukherjee, B., Pesavento, G.: Ethernet passive optical network (EPON): building a next-generation optical access network. IEEE Comm. Mag, Vol. 40, issue. 2 (2002) 66-73

2. Kramer, G., Mukherjee, B., Pesavento, G.: IPACT-a dynamic protocol for an Ethernet PON (EPON). IEEE Comm. Mag, Vol. 40, issue. 2 (2002) 74-80

3. Rege, K., Dravida, S., Nanda, S., Narayan, S., Strombosky, J., Tandon, M., Gupta, D.: QoS Management in Trunk-and-Branch Switched Ethernet Network. IEEE Comm. Mag, Vol. 40, issue. 12 (2002) 30-36

4. Dovrolis, C., Stiliadis, D., Ramanathan, P.: Proportional Differentiated Services: Delay Differentiated and Packet Scheduling. IEEE/ACM Transaction on Networking, Vol.10, issue. 1 (2002) 12-26 